\title{
Electronically Isolated Measuring Circuit for Multicell Traction Battery Modules Identifying State of Charge (SOC) Values
}

\author{
Michal Pipiska, Michal Frivaldsky*, Matus Danko, Jozef Sedo \\ Department of Mechatronics and Electronics, University of Zilina, \\ Zilina, Slovakia \\ michal.frivaldsky@fel.uniza.sk
}

\begin{abstract}
Paper focuses on the topic related to State of Charge (SOC) estimation of the battery modules. The design issues of the electronic circuit suited for very accurate measurements of voltage and current used for evaluation of the SOC parameters of battery module (NexSys $12 \mathrm{~V}$ ) are presented. The SOC evaluation is not described here, but the principles are based on the open-circuit voltage measurement in combination with the coulomb counting method. Both methods are considered within the practical design approach of evaluation circuit of traction lead-acid batteries connected in series ( 3 cells). The circuit proposal is compared to the results of the computer analysis and consequently by the experimental measurement. Mutual comparisons are done for each battery within the module. The results show a very high accuracy between the simulation and measurement, while the relative tolerance varies from $-7 \%$ to $1 \%$ within wide measuring ranges.
\end{abstract}

Keywords-Lead-acid battery; Voltage measurement; Series-parallel connection; SOC; Estimation.

\section{INTRODUCTION}

Current trends within modern applications utilizing energy storage components require individual approach if State of Charge (SOC) estimation is considered. Reflecting on the expansion of industry 4.0 systems, it is expected to be informed about actual power consumption and SOC of battery powered systems, while online information is reported to operator of the industrial internal logistics (inhouse factory dispatching). These mentioned facts are the most important if we discuss about automated infrastructure, i.e. when automated guided vehicles $(\mathrm{AGV})$ are the main part of in-house industrial processes. In order to ensure continuous operation conditions with AGV systems, SOC evaluation is a very important item. If this measurement is reliable and accurate, it opens the possibilities of determining the working radius of the AGV system and planning its continuous replacement, eliminating the disruption of smooth industrial processes and maximizing energy storage system lifetime [1]-[6].

The SOC parameter of the energy storage tank depends on the temperature conditions, the type of storage tank and the

Manuscript received 4 January, 2019; accepted 11 April, 2019.

This research was funded by a grant No. APVV-15/0396 and No. APVV-17/0345 and by a grant No. Vega-1/0119/18. operating conditions. Since these phenomena differ when considering different application areas (energy supply, electromobility, industry), an individual approach is then required for each of these segments with respect to the design of the SOC system. Simultaneously, high-power applications like energy storage systems, automotive applications, industrial automated guided vehicles, and mobile robots require huge battery modules targeting the required voltage for electric drive and for required usable capacity for the active operation [7], [8].

High-end applications require to monitor each battery independently due to manufactural differences within individual battery cells and due to uneven discharge/charge process. For that purpose, proper measuring sub-circuit is required in order to estimate the individual cells' voltage with defined voltage ratio and properties related to the electric isolation.

This paper focuses on an example of practical realization of the measuring circuit, which will be subsequently used to estimate the SOC parameter for the connection of multi-cell, series-parallel configuration of traction lead-acid batteries. The first chapter describes the methods of current and voltage measurement techniques for identification purposes of SOC parameter. Then for a specific battery type, the analysis of output characteristics was carried out in order to determine the equivalent circuit components of the battery. These results are then used to design an accurate electronic measuring circuit for series-connected batteries. The primary design tool of proposed circuit was computer simulation. Accordingly, the measuring circuit must convert the sensed voltages to levels that are allowed for MCU peripherals (SOC evaluation part). At the end of the paper the simulation results are compared with experimental measurements, which were realized by means of a functional sample of the designed system.

\section{MeAsuring Methods}

\section{A. Current-Based Method}

Current-based methods identify the amount of charge remaining in the battery. The principle lies in the measurement of the charge taken from the battery during discharge or supplied to the battery during charge. This 
method is called "coulomb counting". The evaluation of the SOC parameter by means of this method is based on increase or decrease of the charge amount by measuring current within given time intervals. This method is theoretically highly accurate, but if the degradation of components (batteries) is considered, the evaluation error increases.

Since this is a simple and relatively accurate method for evaluation of SOC, its use is relatively noticeable in different application areas. A schematic diagram of the current measurement is shown in Fig 1. The magnitude of the current is evaluated by the electronic circuit from the voltage drop generated by the current flow through the $\mathrm{R}_{\mathrm{SENSE}}$ resistor.

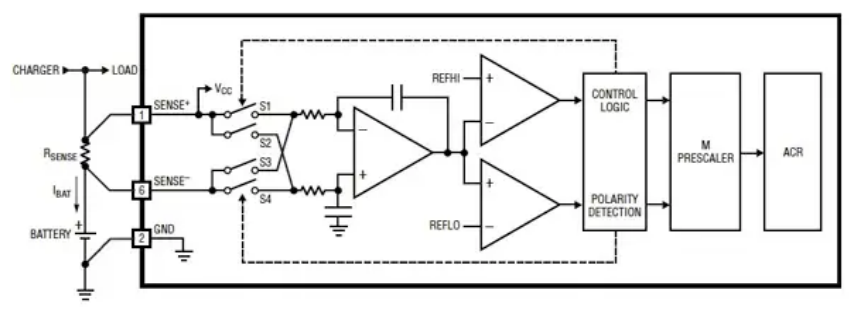

Fig. 1. Principle of current based SOC estimation.

The integrated circuit (IC) applies the differential voltage between SENSE+ and SENSE- to an auto-zeroed differential analog integrator to convert the measured current to charge. Consequently, a host MCU reads the values of the charge and translates it to the so-called Accumulated Charge Register (ACR). This register can be fed by a programmable pre-scaler. The ACR is incremented or decremented by one with each underflow or overflow of the pre-scaler, so that integration time can be effectively scaled. It is also possible to improve such circuitry by implementation of sigma-delta ADC for voltage monitoring that enables to apply voltagebased methods for SOC estimation [9], [10].

\section{B. Voltage-Based Method}

The voltage-based method identifying the SOC parameter monitors actual value of the battery voltage. The voltage is measured when the battery is under load, and after the cell has been disconnected from load, the size of the open circuit battery voltage (OCV) must match the current SOC status. This phenomenon requires special attention, since after each load disconnection from the cell, internal regeneration process of the structure occurs. This process causes large differences of the values considering actual and measured SOC values if insufficient adjustment of the evaluation circuit is provided. The use of a voltage method to determine SOC is also problematic in applications where high dynamics is required, i.e. where rapid load changes apply.

Each of the methods shows some forms of deficiency. Based on this fact, it is advisable to combine the advantages of both methods, which makes it possible to increase the accuracy of SOC evaluation. Coulomb counting method shall be considered for fast dynamic events. On the other hand, in steady-state events, the OCV method of evaluation can be used. Within the application area considered here, a combination of CC and OCV methods will be used based on the above-mentioned facts.

For precise current and voltage measurements, it is necessary to design a proper circuitry, mainly when commercial solutions are not disposable. This is mostly the case of the specific requirements on the battery modules and application. Next, the custom voltage and current monitoring circuit is proposed for the purposes of the SOC estimation within electric drive of the industrial mobile robot. Main parameters and requirements are:

- Battery type: HAWKER NexSys $12 \mathrm{~V}$;

- Battery voltage: 11-13 V;

- Batteries' connection: 3x in series;

- Measured current: $I_{\min }=-100$ A, $I_{\max }=100$ A;

- Evaluation unit: DSP from Texas Instruments.

\section{UtILIZED StATE OF CHARGE Estimation METHOD}

For the SOC estimation of the above-mentioned battery modules, the combination of two techniques was utilized (Current and Voltage methods). For these purposes the measuring circuit was adapted for the custom connection (Fig. 2).

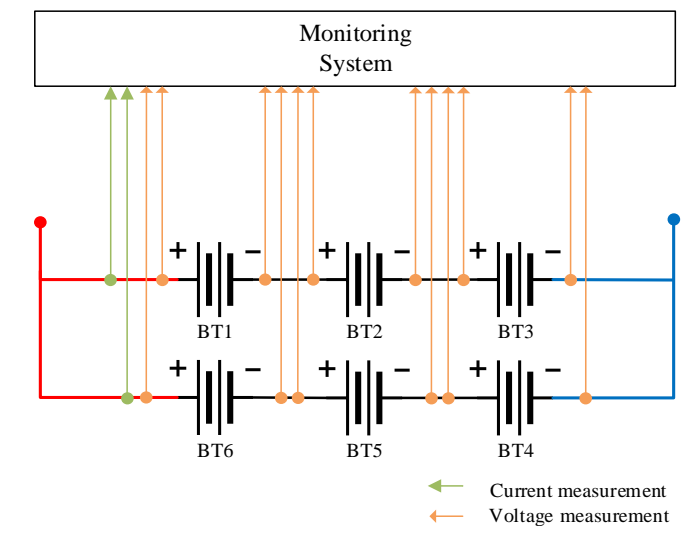

Fig. 2. Battery module and the depiction of current and voltage measurements within individual cells.

Coulomb counting method is a common technique for estimation of a battery SOC, while the current is integrated from the battery. There is a problem, which decrease the accuracy and robustness of this method. It is related to the used current sensor and evaluating unit (MCU), which is limited by the sampling time during the charge or discharge processes of the batteries. Some of the charging units are characterized by the pulse current charging with defined frequency of pulses. If these pulses are too short compared to the sampling performance of the used MCU, then the evaluation of SOC will not be accurate enough. On the other side, this estimation method is good for tracking the rapid changes of SOC. The principle of evaluation is described by (1), while the equation was applied within the estimation circuit

$$
S O C=S O C_{0}+\frac{1}{C} \int_{t_{0}}^{t_{n}}\left(I_{b a t 1}-I_{b a t 0}\right) d t
$$

where $C$ is the rated capacity of battery, $I_{b a t l}$ is the battery current, and $I_{\text {bato }}$ is the battery current by the loss reactions.

An OCV - SOC estimation is suitable mostly for lead-acid batteries. Method is easy to be applied, and even it shows a 
certain amount of inaccuracy, it can be reliable if factors such temperature dependency and output characteristic nonlinearity of battery is considered. Therefore it is recommended to provide the detailed charging and discharging test for the estimation of the output characteristic, from which OCV is defined. These characteristics can differ when new or used battery is considered. Therefore, SOC performance for this method is highly dependent on the actual state of the health of battery used.

For selected types of batteries (HAWKER NexSys 12 V), we have provided a cyclic discharge and charge measurement in order to evaluate the averaged values of OCV (Fig. 3).

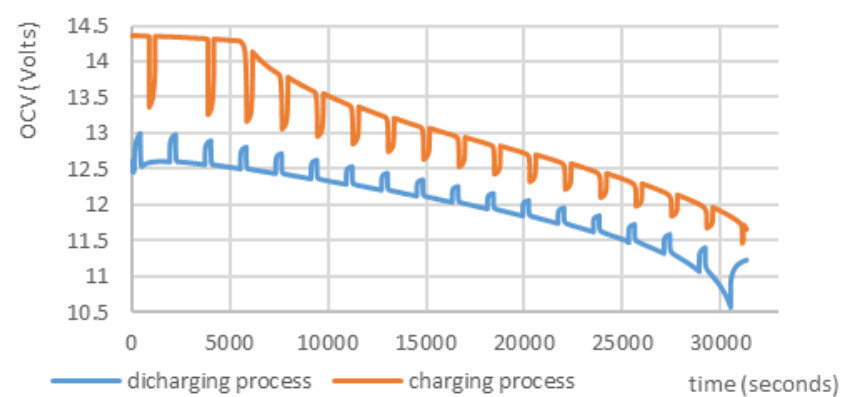

Fig. 3. Output characteristic of HAWKER NexSys $12 \mathrm{~V}$ (12NXS166) for OCV extraction during SOC estimation.

Both methodologies were combined for the target application of SOC estimation. Thus, the main electronic measuring circuit was adapted to this requirement. More detailed description is given below.

\section{Electrical Diagrams of Proposed Measuring CIRCUITS}

The measuring circuit is divided into two parts, one responsible for current measurement and the other for $\mathrm{OCV}$ measurement. For these needs, a hall-sensor ACS758LCB$100 \mathrm{~B}$ is used. The conversion ratio for the evaluation unit, which is DSP from Texas Instruments, is adapted by the R4 and $\mathrm{R} 3$ resistors. The current measurement is responsible for the identification of the amount of charge during the discharge and charge processes, while the OCV method is used as a secondary supporting method for the SOC identification. The detailed circuit for the current measurement of one series string of the battery module is shown in Fig. 4.

The voltage measurement is independent for each battery connected in the module (3-string series connection). The individual operational amplifiers are set for this series connection with respect to the conversion voltage ratio. The measuring circuit also reduces the measured voltage at individual cells of module so that these values are suitable for the peripherals of the evaluation circuit. The wiring diagram is shown in Fig. 5. This is a circuit for one battery of the serial module (with the lowest potential), where common ground with consequent evaluation unit is considered. The remaining circuits have a similar configuration, while the auxiliary resistors set the appropriate measuring voltage level. The most important variable is the voltage conversion ratio and its accuracy for individual cells. For exact identification of the SOC based on the OCV method, it is required to have a very exact voltage measurement of individual battery cells connected in series. The presented application can identify voltage values within the range of 3 decimal points.

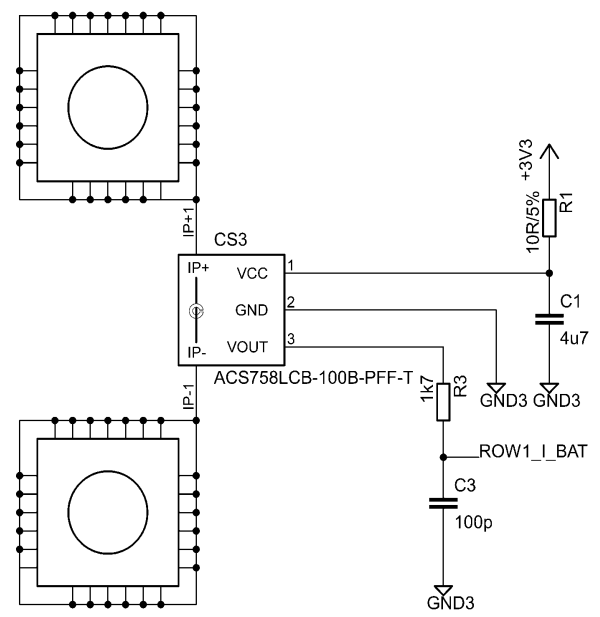

Fig. 4. Measuring circuit for current sensing during charging/discharging processes of the battery.

Another important issue to solve is the design of the circuit, which will be able to identify module's voltages, while just one battery of the module has a common ground with the evaluation unit of SOC. The simplest, but the less accurate way is the use of the voltage dividers connected between the plus cathode of individual batteries and common ground. The difference between the two neighbor dividers reflects the battery voltage. Disadvantage is the fact that, if the battery is connected much above the common ground, the accuracy of the measurement decreases.

The presented proposal is based on the measurement of the voltage of each battery within the range from $11 \mathrm{~V}$ to $15 \mathrm{~V}$ (Fig. 6). For that purpose, exact reference $(10 \mathrm{~V})$ is being created for each battery. Consequently, the differential measurement of the voltage between this reference and plus terminal of the battery is realized. The measured voltage difference is, then, consequently amplified to double these values. The output from the differential amplifier and minus terminal of the battery are connected through the voltage dividers to the common ground. The middle point from the voltage dividers are connected to the voltage followers (buffer amplifier), which are responsible for the impedance isolation between voltage dividers and subsequent circuit. Then, the output from the voltage followers are connected to the inputs of another differential amplifier, which adapts the measured differential voltage to $0,52 \mathrm{~V}\left(\mathrm{U}_{\mathrm{BAT}}=11 \mathrm{~V}\right)$ to $2,659 \mathrm{~V}\left(\mathrm{U}_{\mathrm{BAT}}=15 \mathrm{~V}\right)$, what is necessary for the A/D inputs of the evaluation unit. The evaluation unit, then, recalculates the real OCV value of the battery based on (2)

$$
U_{B A T_{-} X}=10+1.88 * U_{B A T_{-} X_{-} O U T},
$$

where $\mathrm{U}_{\mathrm{BAT}} \mathrm{x}$ is the real value of the OCV of the battery and $\mathrm{U}_{\text {BAT_X_OUT }}$ is the voltage value from the differential amplifier. 


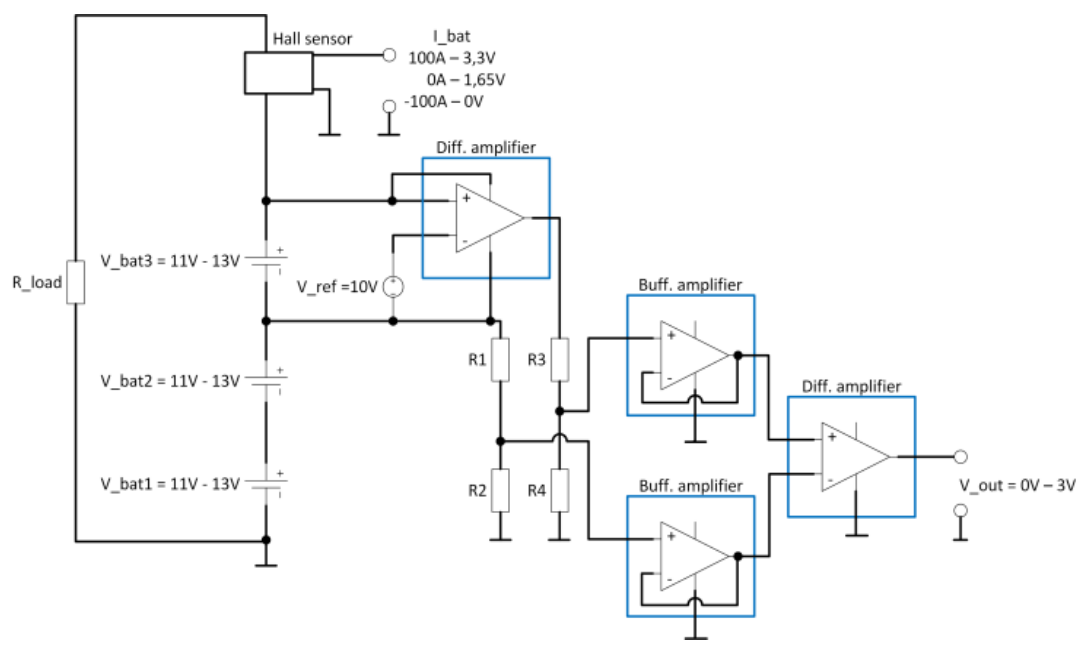

Fig. 5. Block diagram for current and voltage sensing during charging/discharging processes of the battery.

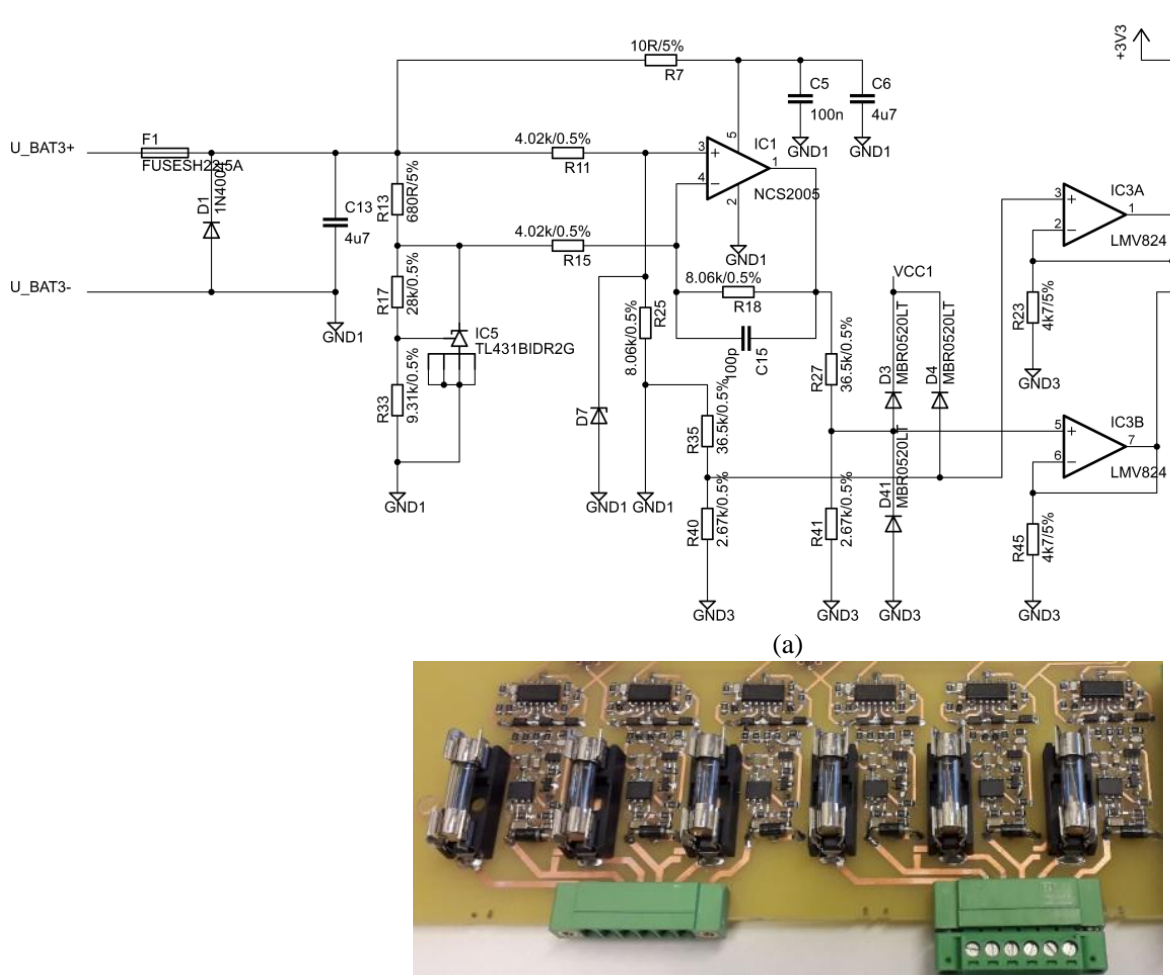

(b)

Fig. 6. Measuring circuit for voltage sensing for OCV identification of (a) one battery from the battery module and (b) experimental testing board.

\section{VERIFICATION OF THE PROPOSED ELECTRONIC MEASURING CIRCUIT}

The verification of the proposed circuit was initially done by the simulation in Matlab SIMULINK (Fig. 7). The battery module is modeled by the equivalent voltage sources what reflects to the OCV of the measured battery. The main investigated variable was the voltage conversion ratio, what is important for the evaluating unit.

Table I shows numerical results from the simulation experiment, whereby the procedure was as follows.

- OCV of each battery ( $\left.\mathrm{U}_{\mathrm{BAT} 1}-\mathrm{U}_{\mathrm{BAT} 3}\right)$ was varying from its upper level $(15 \mathrm{~V})$ - $\mathrm{U}_{\mathrm{BAT}}$ - to its bottom level (11 V).

- The values, which lie between these values are relevant for the SOC estimation. The conversion ratio for individual cells is the same, and voltages U UUT1-U OUT3 are supplied to the evaluation MCU.

The experimental verification was realized similarly to the simulation. The OCV was emulated by the adjustable voltage sources, while the value was changed from top to the bottom value of the battery. Table I shows numerical results from the experimental measurement also. Results show minimal difference between the simulation results and experimental measurements. The evaluated relative error is within whole measured range for each battery. Table II shows numerical results of the relative error (3) for the investigated data, and it is seen that it varies from $-7 \%$ from its maximum to $0 \%$ at minimum

$$
\operatorname{Re} l_{-} E r r o r=\frac{U_{B A T_{-} M E A S}-U_{B A T_{-} S I M}}{U_{B A T_{-} M E A S}} .
$$

The highest error is presented for the top battery from series connection of the module. This highest deviation is related to the requirement on the most precise setting of OP AMPs, which, for this part of the module, converts the 
highest voltage of the module.

interpretation of the measured and simulated values of

Finally, Fig. 8-Fig. 10 are show the graphical voltage conversion ratio.

TABLE I. SIMULATION AND EXPERIMENTAL VALIDATION OF PROPOSED MEASURING CIRCUIT

\begin{tabular}{|c|c|c|c|c|c|c|c|c|c|c|c|}
\hline \multicolumn{6}{|c|}{ Simulation results } & \multicolumn{6}{|c|}{ Real measurement } \\
\hline $\mathbf{U}_{\mathrm{BAT1}}[\mathbf{V}]$ & Uout1 $[\mathbf{V}]$ & $\mathbf{U}_{\text {BAT2 }}[\mathbf{V}]$ & $\mathbf{U}_{\text {out2 } 2}[\mathbf{V}]$ & $\mathbf{U}_{\text {BAT3 }}[\mathbf{V}]$ & Uout3 [V] & $\mathbf{U}_{\mathbf{B A T I}}[\mathbf{V}]$ & UouT1 $[\mathbf{V}]$ & $\mathbf{U}_{\text {BAT2 }}[\mathbf{V}]$ & $\mathbf{U}_{\text {OUT2 } 2}[\mathbf{V}]$ & $\mathbf{U}_{\mathbf{B A T 3}}[\mathbf{V}]$ & $\begin{array}{c}\text { U }_{\text {OUT3 }} \\
{[\mathbf{V}]}\end{array}$ \\
\hline 11 & 0.5239 & 11 & 0.5242 & 11 & 0.5244 & 11.004 & 0.488 & 11.015 & 0.534 & 11.017 & 0.53 \\
\hline 11.5 & 0.7911 & 11.5 & 0.7915 & 11.5 & 0.7918 & 11.503 & 0.752 & 11.497 & 0.795 & 11.507 & 0.789 \\
\hline 12 & 1.0584 & 12 & 1.0589 & 12 & 1.0592 & 12.002 & 1.017 & 11.992 & 1.055 & 12.004 & 1.053 \\
\hline 12.5 & 1.3256 & 12.5 & 1.3262 & 12.5 & 1.3266 & 12.502 & 1.282 & 12.514 & 1.334 & 12.506 & 1.319 \\
\hline 13 & 1.5928 & 13 & 1.5936 & 13 & 1.5941 & 13.002 & 1.547 & 13.048 & 1.619 & 12.996 & 1.579 \\
\hline 13.5 & 1.86 & 13.5 & 1.8609 & 13.5 & 1.8615 & 13.499 & 1.809 & 13.52 & 1.871 & 13.494 & 1.846 \\
\hline 14 & 2.1272 & 14 & 2.1282 & 14 & 2.1289 & 14 & 2.075 & 14 & 2.127 & 14.042 & 2.138 \\
\hline 14.5 & 2.3944 & 14.5 & 2.3956 & 14.5 & 2.3963 & 14.502 & 2.341 & 14.502 & 2.394 & 14.522 & 2.395 \\
\hline 15 & 2.6615 & 15 & 2.663 & 15 & 2.6638 & 15 & 2.605 & 15.055 & 2.687 & 15.017 & 2.659 \\
\hline
\end{tabular}

TABLE II. EVALUATION OF THE RELATIVE ERROR BETWEEN MEASUREMENT AND SIMULATION

\begin{tabular}{|l|c|c|c|c|c|c|c|c|c|}
\hline \multicolumn{10}{|c|}{ Relative error evaluation } \\
\hline UOUT_REL1 & $-7 \%$ & $-5 \%$ & $-4 \%$ & $-3 \%$ & $-3 \%$ & $-3 \%$ & $-3 \%$ & $-2 \%$ & $-2 \%$ \\
\hline UOUT_REL2 & $2 \%$ & $0 \%$ & $0 \%$ & $1 \%$ & $2 \%$ & $1 \%$ & $0 \%$ & $0 \%$ & $1 \%$ \\
\hline UOUT_REL3 & $1 \%$ & $0 \%$ & $-1 \%$ & $-1 \%$ & $-1 \%$ & $-1 \%$ & $0 \%$ & $0 \%$ & $0 \%$ \\
\hline
\end{tabular}

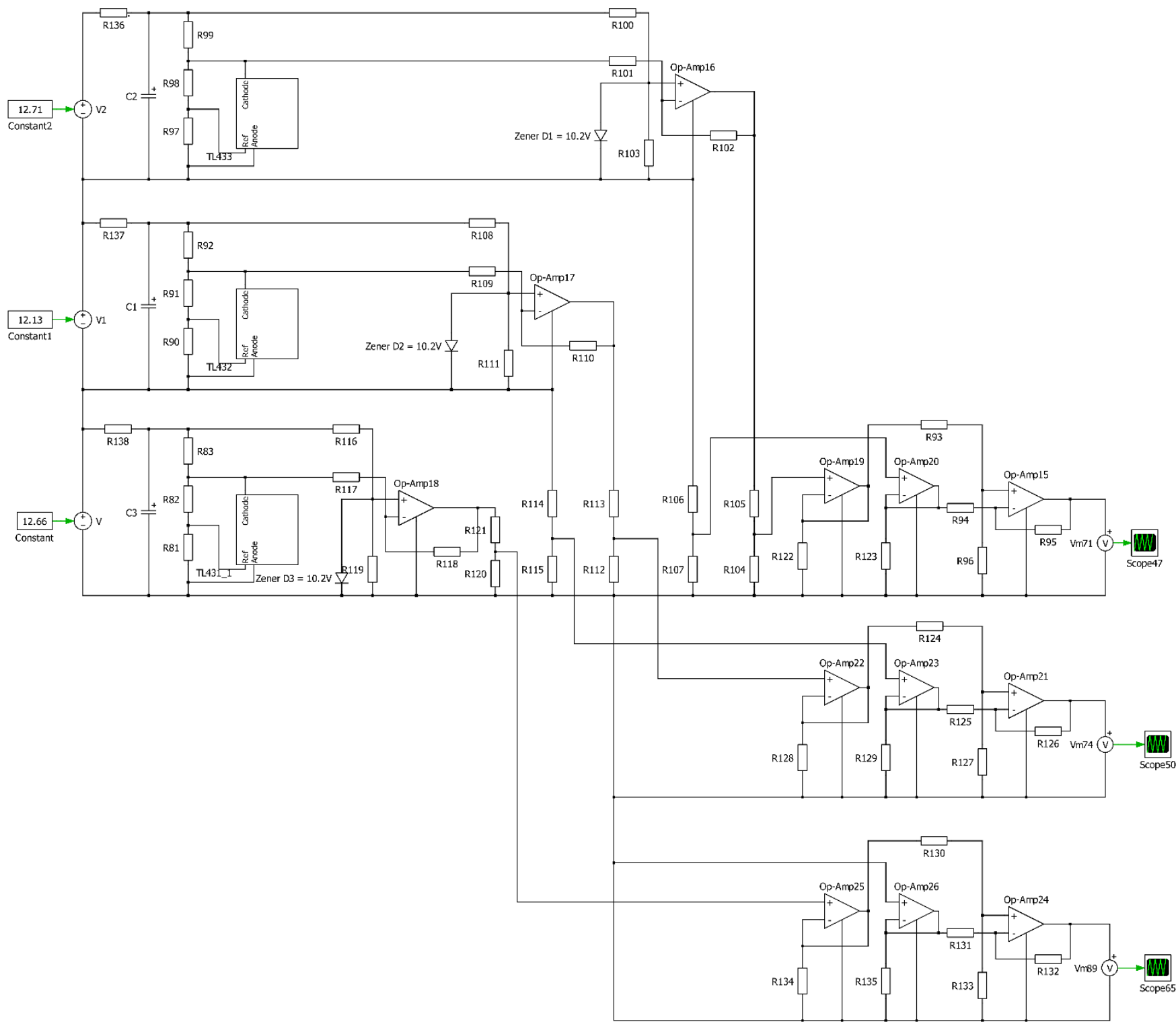

Fig. 7. Simulation model of the measuring circuit for voltage sensing. 
For individual batteries relevant results are compared, whereby it is seen that evaluated difference corresponds to the evaluated data of the relative error. Thus, the highest visible difference is for the case of Battery 1, which is connected at the highest potential of the battery module. As a summary it could be said that designed measuring system of voltage battery level identification provides required functionality and is suitable for the purposes related to target application, which is the SOC estimation of the considered battery module.

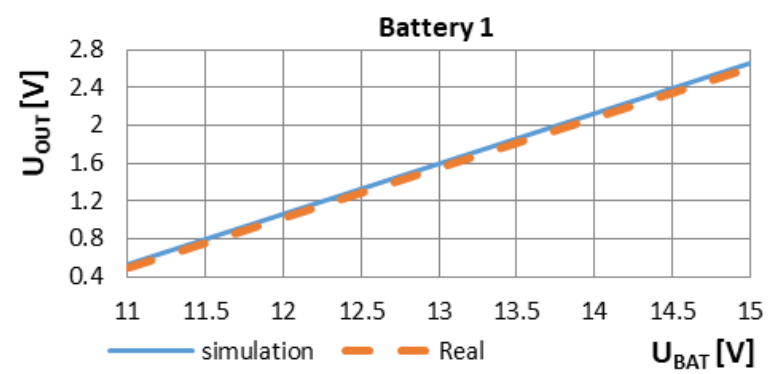

Fig. 8. Evaluation of battery voltage (battery_1) in form of converted voltage at the output of measuring circuit.

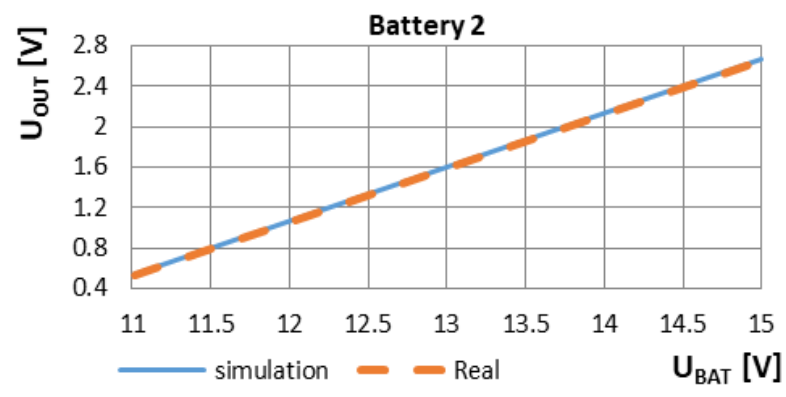

Fig. 9. Evaluation of battery voltage (battery_2) in form of converted voltage at the output of measuring circuit.

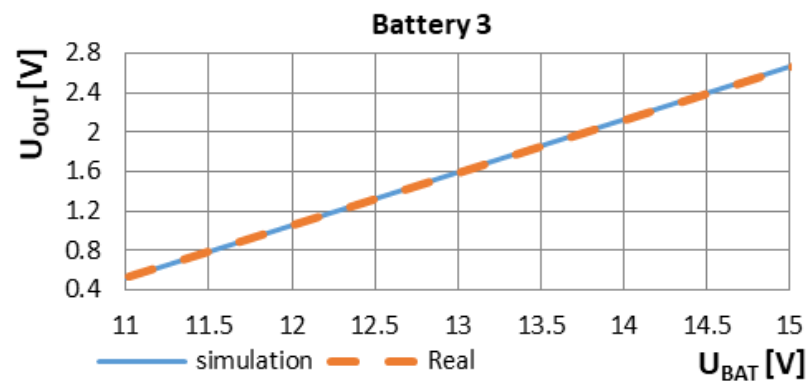

Fig. 10. Evaluation of battery voltage (battery_3) in form of converted voltage at the output of measuring circuit.

\section{CONCLUSIONS}

Within proposed paper a possible way for design of the electronic circuit for OCV (Open-Circuit Voltage) estimation of the traction battery is provided. Designed circuit represents interface between the measured component and evaluation system (DSP). Key solution for precise measurement of OCV was required for series connection of 3 batteries. Instead of that relevant conversion voltage ratio of the battery voltages and output voltages sourced to the evaluating processor was required. The circuit design uses operational amplifiers (differential types) connected in cascade topologies. Each cell of the battery module has its own measuring channel. The proposal was evaluated by the simulation analysis, first, where proper functionality and component calculation was confirmed. Consequently, practical design was realized and verified. Experimental measurements showed that the difference between the expected (simulation) and real (measurements) behavior vary within the range of the relative error in interval from $7 \%$ to $0 \%$. The highest deviation was found for the top cell connected at the highest potential of series batteries connection. Due to this fact, the proposed system has to convert higher voltage for this battery with the lower accuracy compared to the others. Component tolerance value for the experimental measuring sample influenced the measuring results also. Finally, it can be said that the proposed measuring circuit exhibits the required performance for SOC and can be further used for the evaluation units, whose input voltages ranges from $0 \mathrm{~V}$ to $3,3 \mathrm{~V}$.

\section{CONFLICTS OF INTEREST}

The authors declare that they have no conflicts of interest.

\section{REFERENCES}

[1] J. Sabatiera, M. Aouna, A. Oustaloupa, G. Grégoireb, F. Ragotb, and P. Royb, "Fractional system identification for lead acid battery state of charge estimation", Signal Processing, vol. 86, no. 10, pp. 2645 2657, 2006. DOI: 10.1016/j.sigpro.2006.02.030.

[2] G. Nobile, M. Cacciato, G. Scarcella, and G. Scelba, "Multi-criteria experimental comparison of batteries circuital models for automotive applications", Communications, vol. 20, no. 1, pp. 97-104, Mar. 2018.

[3] C. Cai, D. Du, Z. Liu, and J. Ge, "State-of-charge (SOC) estimation of high power Ni-Mh rechargeable battery with artificial neural network", in Proc. of the 9th International Conference on Neural Information Processing (ICONIP'OZ), 2002. DOI: 10.1109/ICONIP.2002.1198174.

[4] S. Piller, M. Perrin, and A. Jossen, "Methods for state-of-charge determination and their applications", Journal of Power Sources, vol. 96, pp. 113-120, 2011. DOI: 10.1016/S0378-7753(01)00560-2.

[5] G. Aiello, M. Cacciato, S. Messina, and M. Torrisi, "A high efficiency interleaved PFC front - end converter for EV battery charger”, Communications, vol. 20, no. 1, pp. 86-91, Mar. 2018.

[6] S. Rodriguesa, N. Munichandraiahb, and A. K. Shuklaa, "A review of state-of-charge indication of batteries by means of a.c. impedance measurements", Journal of Power Sources, vol. 87, no. 1-2, pp. 1220, 2000. DOI: 10.1016/S0378-7753(99)00351-1.

[7] R. Bayir and E. Soylu, "Real time determination of rechargeable batteries' type and the state of charge via cascade correlation neural network", Elektronika Ir Elektrotechnika, vol. 24, no. 1, 2018. DOI: 10.5755/j01.eie.24.1.20150

[8] F. Huet, "A review of impedance measurements for determination of the state-of-charge or state-of-health of secondary batteries", Journal of Power Sources, vol. 70, no. 1, pp. 59-69, 1998. DOI: 10.1016/S0378-7753(97)02665-7.

[9] M. Galád, P. Špánik, M. Cacciato, and G. Nobile, "Analysis of state of charge estimation methods for smart grid with VRLA batteries", Electr. Eng., vol. 99, no 4, pp. 1233-1244, 2017. DOI: 10.1007/s00202-017-0618-z.

[10] M. Galád, P. Špánik, M. Cacciato, and G. Nobile, "Comparison of common and combined state of charge estimation methods for VRLA batteries", in Proc. of 2016 ELEKTRO, Strbske Pleso, 2016, pp. 220225. DOI: 10.1109/ELEKTRO.2016.7512069. 